\title{
An Examination of Adolescent Athletes and Nonathletes on Baseline Neuropsychological Test Scores
}

\author{
Christopher P. Tomczyk, LAT, ATC; Megan Mormile, ATC; \\ Megan S. Wittenberg; Jody L. Langdon, PhD; Tamerah N. Hunt, PhD, ATC, FACSM
}

\author{
Department of Health Sciences and Kinesiology, Georgia Southern University, Statesboro
}

Context: An estimated 15.3 million adolescent students are enrolled in US high schools, with approximately 7.8 million participating in athletics. Researchers have examined various demographics in high school athletes; however, athletic participation may play a larger role in test performance than previously thought. Currently, investigations of concussion assessment may rely on uninjured athletes as controls. However, due to the intense nature of athletics, this may not be an appropriate practice.

Objective: To examine differences between athletes and nonathletes using a common computerized neuropsychological test.

Design: Retrospective cross-sectional study.

Setting: High schools from a school district in Columbus, Ohio.

Patients or Other Participants: A total of 662 adolescent high school students (athletes: $\mathrm{n}=383$, female $\mathrm{n}=18$; nonathletes: $n=279$, female $n=193$ ).

Main Outcome Measure(s): Participants were administered a computerized neuropsychological test battery (Immediate
Post-Concussion Assessment and Cognitive Test [ImPACT]) during baseline concussion assessment. Differences between groups were established for output composite scores.

Results: Differences were found between athletes and nonathletes in composite reaction time $\left(F_{1,522}=14.855, P<\right.$ $.001)$ and total symptom score $\left(F_{1,427}=33.770, P<.001\right)$. Nonathletes reported more symptoms, whereas athletes had faster reaction times. No differences were present in composite verbal memory, composite visual memory, composite visual motor speed, or composite impulse control $(P>.05)$.

Conclusions: Symptom reporting and reaction time differed between high school athletes and nonathletes. Participation in extracurricular activities may lead to cognitive differences in adolescents that can influence performance on the Immediate Post-Concussion Assessment and Cognitive Test battery. Researchers should account for these differences in baseline performance when making concussion diagnostic and management decisions.

Key Words: concussions, youths, sports

\section{Key Points}

- At baseline, adolescents' athletic status appeared to influence symptom reporting and reaction time.

- Due to the potential influence of athletic status, we recommend, when possible, not relying on nonathletes as healthy controls in future concussion research.

$\mathrm{S}$ ince 1989, the population for concussion-based research has been almost exclusively athletes, primarily because of their high rate of injury. Approximately 63000 concussions occur annually in the US high school population, with the incidence rate estimated to be as high as $15.3 \% .{ }^{1,2}$ Concussion, commonly defined as a complex pathophysiological process affecting the brain caused by either direct or impulsive biomechanical forces, is accompanied by a varied manifestation of symptoms, altered cognition, and postural instability, making every patient unique. ${ }^{3,4}$ Due to the unpredictability of concussion symptoms, evidence-based position statements have recommended a multifaceted approach for managing all patients with concussions, regardless of severity. ${ }^{1-4}$ This has led to the implementation of specific recommendations, including cognitive rest, symptom monitoring, and neuropsychological testing, for all patients with concussion. ${ }^{3-6}$ Each intervention is implemented to address a certain aspect of concussion and neuropsychological testing measures cognitive ability.

Neuropsychological tests are designed to measure psychological functions that have been linked to a specific brain function or pathway. ${ }^{7}$ In concussion management, neuropsychological tests are used to detect differences in cognitive ability and have been shown to be more reliable when compared with baseline data. ${ }^{6}$ For example, normative population data were reliable in detecting these cognitive deficits, although baseline testing has typically been used most often. ${ }^{6}$ Baseline testing to assess the premorbid state of cognitive function in an athlete provides the individual's scores for comparison in case of a concussive injury., ${ }^{4,6}$ Baseline testing allows for better detection of neurocognitive deficits in the individual athlete. ${ }^{6}$

The presence of neurocognitive deficits is a key component of concussion diagnosis and heavily influences 
the management process. Therefore, correctly interpreting the results of neuropsychological testing is paramount. The Immediate Post-Concussion Assessment and Cognitive Test (ImPACT; ImPACT Applications Inc, San Diego, CA) is a computerized neuropsychological battery widely used in the high school setting. Upward of $90 \%$ of all high school athletic trainers (ATs) who used a computerized cognitive battery in their concussion protocol administered the ImPACT. ${ }^{8}$ With the potential for millions of adolescent athletes to be exposed to a particular neuropsychological battery, it is pertinent to examine the performance of different subsets of the high school population. By doing so, we can identify and account for differences in cognitive ability, thereby enhancing the accuracy of interpreting results and making proper diagnostic and management decisions.

Athletic status has not previously been thought to affect neuropsychological testing, but with the potential for high school students from a wide array of backgrounds to take the ImPACT, variables such as athletic status warrant study. Compared with the athletic population, concussion incidence rates in the US nonathletic population are far lower, around 1.4 million a year; the majority result from motor vehicle accidents and falls but are still prevalent enough to warrant investigation. ${ }^{9}$ Considering a high school setting, athletic status is of interest because more than half of the population is categorized as nonathletes. ${ }^{10,11}$ Stressors may differ between athletes and nonathletes based on activity, which may lead to differences in brain development. Given that much athletic participation occurs during adolescence, while the brain is still developing, examining the effect of athletic status on a developing brain may prove important.

The consensus in the literature is that the primary, secondary, and tertiary brain regions are fully developed by age 12 , but cognitive maturity continues to evolve throughout adulthood, especially in the prefrontal cortex. ${ }^{12}$ The continual development of the brain throughout childhood and into adolescence may differ depending on the activities and environment to which a person is exposed. Multiple neuroimaging studies ${ }^{13,14}$ have shown developmental differences in adolescent brains, controlled by factors including socioeconomic status and participation in extracurricular activities (eg, music). This may have a direct effect on neuropsychological testing, based on the possibility of structural differences of the brain pathways targeted by the neuropsychological tests themselves. With the possibility of fundamental structural differences among adolescents based on athletic status, it is essential to understand how the performance of these 2 groups compares on commonly used computerized neuropsychological tests. Another potential benefit of examining the effects of athletic status on neuropsychological test performance is the possibility of studying nonathletes as matched controls in future research. It is common practice in concussion research to rely on other athletes as matched controls, which can be limited by accessibility, although without examination of athletic status as an influencing variable, it may be unwise to make such comparisons.

Concussions are complex injuries that are prevalent in both the athlete and nonathlete high school populations. The standard of care for concussion has been defined by position statements and includes the use of neuropsycho- logical testing to detect and monitor cognitive deficits. Previous authors have demonstrated that exposure to the ImPACT is widespread, resulting in the need to examine variables that may influence performance. Furthermore, these potential cognitive differences at baseline need to be accounted for when making clinical decisions. Therefore, the purpose of our study was to examine the effects of athletic status (athlete versus nonathlete) on baseline neuropsychological test scores in a high school population in order to facilitate more accurate interpretations of the results.

\section{METHODS}

\section{Study Design}

The design was retrospective, cross-sectional, and between groups.

\section{Participants}

A sample of 662 high school students participated in this study. Participants were students (athlete $\mathrm{n}=383$, age $=$ $15.76 \pm 1.14$ years, female $n=18$; nonathlete $n=279$, age $=15.28 \pm 1.22$ years, female $n=193$ ) from a single school district in Columbus, Ohio. Recruitment involved all interscholastic sports and clubs (ie, marching band, physical education courses, health courses and clubs) across both sexes (height: males $=177.50 \pm 9.43 \mathrm{~cm}$, females $=162.21 \pm 9.75 \mathrm{~cm}$; weight: males $=80.65 \pm$ $22.86 \mathrm{~kg}$, females $=63.46 \pm 14.71 \mathrm{~kg}$ ). All participants and their guardians read and signed informed assent and consent forms, respectively, approved by the institutional review board (which also approved the investigation) before the study began. Exclusion criteria were incomplete data on athletic status, a self-reported history of concussion, a musculoskeletal injury in the 3 months before testing, English as a second language, or a self-reported diagnosis of a psychiatric or learning disability. Nonathletes were not questioned about athletic activity outside of school (eg, private clubs or lessons); therefore, involvement in extracurricular athletics was not an exclusionary factor. All participants were required to complete a computerized neuropsychological test (ImPACT) as a baseline assessment.

\section{Neuropsychological Battery: ImPACT}

The ImPACT is an Internet-based computerized assessment that has become one of the most widely used neuropsychological batteries. ${ }^{4,6,8,15}$ The instrument contains 3 domains of concussion assessment (demographics, symptoms, and neuropsychological testing) with 6 measures that yield 5 quantitative composite scores: verbal memory, visual memory, visual-motor speed (processing speed), reaction time, and impulse control. ${ }^{15,16}$ The ImPACT yields 5 quantitative composite scores: verbal memory, visual memory, visual motor speed, composite reaction time, and composite impulse control. Researchers $^{15,17,18}$ have supported ImPACT as a valid measure for neurocognitive deficits after sport-related concussion, especially when compared with a baseline. Although ImPACT is a valid construct for concussion assessment and yields high sensitivity $(91.4 \%)$, specificity has been 
Table 1. Demographic Values for Adolescent Athletes and Nonathletes

\begin{tabular}{|c|c|c|}
\hline Characteristic & Athletes $(n=383)$ & Nonathletes $(n=279)$ \\
\hline \multirow[t]{2}{*}{ Females, $\mathrm{n}$} & 18 & 193 \\
\hline & \multicolumn{2}{|c|}{ Mean $\pm S D$} \\
\hline Age, y & $15.76 \pm 1.14$ & $15.28 \pm 1.22$ \\
\hline Height, cm & $177.14 \pm 9.95$ & $166.42 \pm 11.57$ \\
\hline Weight, kg & $80.88 \pm 23.09$ & $67.35 \pm 17.96$ \\
\hline
\end{tabular}

only moderate $(69.4 \%) .{ }^{18}$ The ImPACT battery also can be administered in a group setting without decreasing the validity of each composite score. ${ }^{19}$ Overall, due to ImPACT's validity, convenience of administration, and heightened popularity among high schools, it was the ideal neuropsychological battery to examine.

\section{Procedures}

We notified the school district and obtained authorization from the administration to conduct this study before any participants were contacted. Consent forms were distributed to each participant's parent or guardian. Once consent was received, minor assent forms were completed, and the participants were enrolled in the study. All participants and their parent or guardian were assured that involvement in the study would not interfere with academic, extracurricular, or athletic status and was strictly voluntary. Participants were also informed that they could withdraw from the study at any time with no repercussions.

All participants were administered the ImPACT battery before the start of the competitive season for athletes and during the school year for nonathletes. Properly trained ATs and researchers administered ImPACT, which takes approximately 30 minutes to complete. The testing took place in the school's computer laboratory with at least 2 chair spaces between participants. In the demographics section, participants were asked to identify themselves as an athlete or a nonathlete. Testing was organized by team and club or organization; therefore, both athletes and nonathletes were tested primarily in a group setting.

\section{Statistical Analysis}

Descriptive statistics were conducted on demographic variables and composite scores. A 1-way analysis of variance was performed to measure differences between athletes and nonathletes for the following composite ImPACT variables: verbal memory, visual memory, visual motor speed, reaction time, impulse control, and total symptom score. We used SPSS (version 23.0; IBM Corp, Armonk, NY) for all analyses. Alpha levels were set a priori at $P<.05$ and adjusted using a Bonferroni correction to a new $\alpha$ level of $P<.0083$.

\section{RESULTS}

Descriptive statistics are reported in Table 1. A 1-way analysis of variance revealed differences between athletes and nonathletes for composite reaction time (athletes $=0.62$ \pm 0.09 , nonathletes $=0.65 \pm 0.11 ; F_{1,522}=14.855, P<$ $.001, r=-0.152$, Cohen $\mathrm{d}=-0.308$ ), and total symptom score $($ athletes $=6.25 \pm 9.00$, nonathletes $=12.03 \pm 14.73$; $F_{1,427}=33.770, P<.001, r=-0.230$, Cohen $\left.\mathrm{d}=-0.473\right)$.
Table 2. Individual ImPACT Composite Scores for Adolescent Athletes and Nonathletes (Mean \pm SD)

\begin{tabular}{lcc}
\hline Composite Score & Athletes & Nonathletes \\
\hline Verbal memory & $79.16 \pm 11.86$ & $81.15 \pm 11.44$ \\
Visual memory & $68.71 \pm 14.18$ & $67.72 \pm 14.09$ \\
Visual motor & $32.82 \pm 7.26$ & $32.81 \pm 7.62$ \\
Reaction time, s & $0.62 \pm 0.09^{\mathrm{a}}$ & $0.65 \pm 0.11^{\mathrm{a}}$ \\
Impulse control & $7.66 \pm 6.19$ & $7.31 \pm 5.87$ \\
Total symptom score & $6.25 \pm 9.00^{\mathrm{a}}$ & $12.03 \pm 14.73^{\mathrm{a}}$ \\
\hline
\end{tabular}

Abbreviation: ImPACT, Immediate Post-Concussion Assessment and Cognitive Test (ImPACT Applications, Inc, San Diego, CA).

a Denotes significance at a priori $P<.0083$.

Due to violation of homogeneity of variance, we computed the Welch $F$ to determine if the differences between groups were significant for both reaction time and symptom score $(P<.001)$. No differences were observed between athletes and nonathletes in composite verbal memory $\left(F_{1,660}=\right.$ 4.653, $P=.031, r=-0.085$, Cohen $\mathrm{d}=-0.170)$, composite visual memory $\left(F_{1,660}=0.794, P<.373, r=0.034\right.$, Cohen $\mathrm{d}=0.070)$, composite visual motor speed $\left(F_{1,660}=0.000, P\right.$ $<.987, r=0.000$, Cohen $\mathrm{d}=0.001)$, or composite impulse control $\left(F_{1,660}=0.794, P<.373, r=0.028\right.$, Cohen $\mathrm{d}=$ 0.058 ; Table 2).

\section{DISCUSSION}

The purpose of our study was to examine differences between high school athletes and nonathletes on a computerized neuropsychological battery in a baseline assessment. We found differences between athletes and nonathletes for reaction time and symptoms. In athletics, reaction time may determine winning or losing and has been a topic of research globally. In psychometric psychology, reaction time is used as an index of processing speed. ${ }^{20}$ It was not surprising that our data revealed a faster reaction time in the athletes compared with the nonathletes (Figure 1). An ample body of literature has reported similar findings. Bruzi et $\mathrm{al}^{21}$ examined the reaction times of basketball players, gymnasts, and nonathletes. Their testing indicated a slower reaction time in the nonathlete group compared with both athlete groups. These results can be

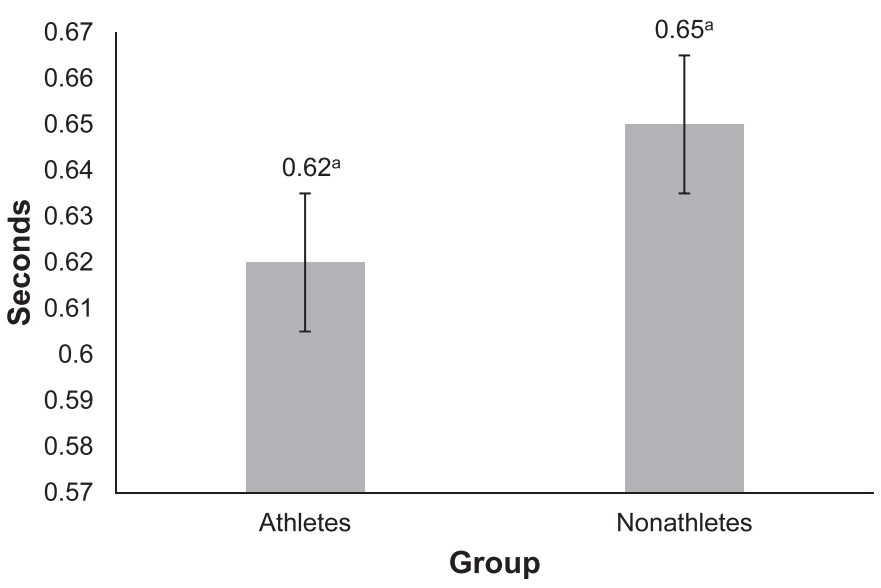

Figure 1. Average composite reaction time scores (mean \pm standard deviation) for adolescent athletes and nonathletes. a Indicates difference. 


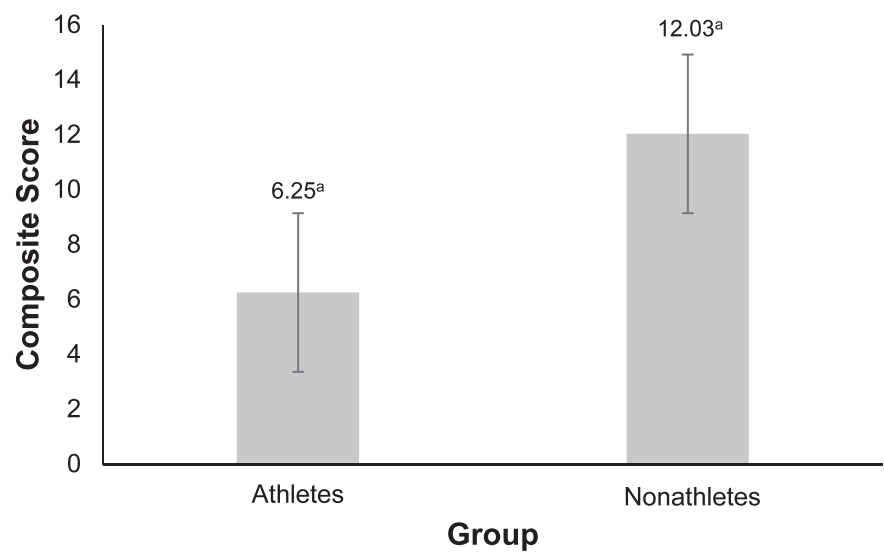

Figure 2. Average total symptom score (mean \pm standard deviation) for adolescent athletes and nonathletes. ${ }^{a}$ Indicates difference.

attributed to the effect of systematic athletic practice in improving reaction times. ${ }^{22-24}$

Additionally, Bruzi et $\mathrm{al}^{21}$ found virtually no difference in reaction time between the 2 athlete groups. This raises the question of whether reaction time is a sensitive enough measure to vary by level of competition. When the influence of athletic skill on reaction time was addressed, results were conflicting. Some authors have indicated that the skill level of the athlete had no effect on reaction time. For example, Balko et $\mathrm{al}^{25}$ observed no difference between the reaction times of elite and nonelite fencers. In soccer players, reaction time was not different among elite and nonelite athletes ${ }^{26}$ Conversely, Williams and Walmsley ${ }^{27}$ concluded that elite fencers had faster reaction times than lower-level fencers. We did not examine differences between levels of athletic competition, but the literature suggests that adolescent athletes at different levels of competition (eg, varsity and junior varsity) should not have differences in reaction time. The vigorous training high school athletes undergo appears to have a significant effect on reaction time, leading to faster scores on ImPACT compared with their nonathlete counterparts. To make proper assessment and management decisions, clinicians should take all of these factors regarding reaction time into consideration when interpreting results for both populations.

The intense nature of athletics also has an effect on symptoms. Like reaction time, total symptom score differed between athletes and nonathletes (Figure 2). The ImPACT total symptom score is based on the total number of symptoms and the severity of each. Therefore, at baseline, athletes reported either fewer concussion symptoms or less severe symptoms (or both) than nonathletes. Several psychosocial factors could lead to this pattern of decreased symptom scores at baseline in athletes, including athletic identity, the culture of risk in sports, and perceived pain tolerance.

Athletic identity is defined as the degree to which an athlete identifies with the athlete role. ${ }^{28}$ Adolescents have been shown $n^{29,30}$ to identify highly with their extracurricular activities (eg, sports), and removal from participation can lead to negative social and psychological effects for the individual. Athletic identity is often intertwined with the culture of risk in sports, which states that athletes must and will accept the risk of injury and sometimes push their bodies past their limits. ${ }^{31}$ The culture of risk in sports normalizes not only their pain but also injury, leading to a play-at-all-costs mentality in athletes. This desensitized state in sports can lead to guilt, shame, and frustration when complaining about pain or injury. ${ }^{32}$ Because ImPACT uses total symptoms reported to calculate a raw score, the results may be skewed if athletes instinctively omit symptoms due to these psychosocial constructs.

Another explanation for why athletes consistently report fewer symptoms at baseline than their nonathlete counterparts is their perception of pain itself, specifically the potential for heightened pain tolerance. Ryan and Kovacic ${ }^{33}$ compared contact athletes, noncontact athletes, and nonathletes and found that contact athletes had more experience with pain and so were able to tolerate more, due to the ability to distinguish if the pain was truly harmful in nature. This may be a factor in the culture of risk in sports and sway symptom reporting, leading to decreased scores in the athletic population, especially those in contact sports. The psychosocial state of adolescent athletes may play a primary role in why we found consistently lower symptom scores in the athlete population compared with the nonathlete population, who may not have been exposed to these factors. The clinician should be aware that athletes may suppress symptoms or symptom severity when selfreporting.

No differences were present between athletes and nonathletes for composite verbal memory, composite visual memory, composite visual motor speed, and composite impulse control (Table 2). Although differences between the groups for composite verbal memory were nonsignificant, a trend suggested that nonathletes scored higher than athletes (81.15 and 79.16, respectively). Previous research$\mathrm{ers}^{34-38}$ have demonstrated variations in verbal memory capacity among adolescents who participated in different activities, specifically music. This could indicate cognitive differences in adolescents based on extracurricular tasks, but more study is needed.

We found that visual memory, unlike verbal memory, appeared to be more consistent between athletes and nonathletes. Although this is not surprising, the mean scores for composite visual memory were close (athletes $=$ 68.71 , nonathletes $=67.72$ ). Multiple investigators ${ }^{35,38}$ noted that visual memory did not increase in adolescents who participated in music (a nonathletic extracurricular activity) versus those who did not, which is in line with the fairly similar performances on composite visual memory we observed.

On ImPACT, composite impulse control is an indication of effort level and is typically monitored to ensure validation of baseline scores. Even though the findings were nonsignificant, athletes had consistently higher composite impulse control scores than their nonathlete counterparts (7.66 and 7.31, respectively). It has been suggested that 1 of every 10 high school football athletes will exhibit poor effort on neuropsychological batteries, and these relatively high impulse control scores suggest the same pattern. ${ }^{39}$ Nonathletes presented with similar scores, which may suggest that adolescents in general put forth similar efforts in baseline testing.

The scores for composite visual motor speed were nearly identical between athletes and nonathletes (32.82 and 
32.81, respectively). Relatively little research has focused on the effects of participation in certain activities on visual motor capabilities in the adolescent population. Our results indicated that participating in athletics had little to no effect on a high school student's ability to perform visual motor tasks.

The exploration of differences between athletes and nonathletes for concussion management is the next logical progression in the literature. As previously mentioned, cognitive ability varies markedly with regard to how it is used in clinical practice. To our knowledge, we are the first to examine the effect of athletic status on baseline neuropsychological test scores, adding to the preexisting body of adolescent concussion literature. This information is vital to clinicians who provide care to adolescents, specifically high school ATs. More than half of the high school population consists of nonathletes, and ATs are increasingly treating nonathletes, specifically band members, cheerleaders, and members of club sports. Some concussion protocols address treatment procedures for nonathletic organizations that often associate with athletic teams.

When a high school AT is faced with managing a concussion in a nonathlete, the protocol normally follows a similar trajectory as for an athlete, including neuropsychological testing. In most of these cases, no baseline test performance will be available. This can become problematic when attempting to compare postinjury ImPACT scores with baseline scores, whether they be for matched peers or normative data. Our findings showed that such comparisons would be invalid and could lead to improper management of the patient's injury. This idea can also be a factor when a clinician is faced with an athlete who has a condition that is not examined during baseline neuropsychological testing. If specific matched comparisons are not available in the athlete population, it is not appropriate to use the data of nonathletes.

The current study adds support to the concept that nonathletes should not serve as matched controls in concussion research. Studying nonathletes as controls in concussion research may be appealing due to a significant increase in potential matches. Additionally, when uninjured athletes function as controls, the inherent risk of injury could lead to possible experimental mortality. A possible but irresponsible solution is nonathlete controls. The potential benefits do not outweigh the inherent limitations that influence the interpretation of data. Along with others ${ }^{40}$ we demonstrated differences in the major tiers of concussion management (symptoms, neuropsychology, and balance) between these 2 populations. Therefore, we recommend not relying on nonathletes as a comparison group in future research.

Our study had several limitations. One limitation was the inability to examine differences in the nonathlete group by activity. We did not examine specific activities in which the nonathlete group participated. Doing so would have allowed for specialized examination of the results and may have offered insight into extracurricular activities that may have had a larger effect on neuropsychological testing. A second limitation was the uneven distribution of males and females within each group. Previous authors ${ }^{41}$ have shown sex-based differences on neuropsychological tests in the adolescent population. Lastly, we investigated a retrospective sample of adolescents in a single school district; therefore, the results may not be generalizable to the entire adolescent population.

\section{CONCLUSIONS}

The present study was among the first to examine differences between the adolescent athlete and nonathlete populations on ImPACT in order to better understand if performance differences were present. Our results can assist clinicians in interpreting results, as well as shedding light on future research involving the 2 populations. After analyzing the results of the present study, we advise that nonathletes not serve as healthy controls or comparison groups in future research. The differences between the 2 groups at baseline were significant and would pose an inherent limitation for any study. The benefit of having more participants for the control group, along with the decreased risk of experimental mortality, does not outweigh the fact that subtle differences could lead to skewed data. Future investigators should, however, continue to explore the role of athletic status in concussion. Authors should address the limitations of the current study pertaining to the effects of specific extracurricular activities on the ImPACT. Additionally, the recovery trajectories and return-to-learn paradigms of nonathletes need to be examined. Concussion is a relative risk for nonathletes; therefore, clinicians may be asked to treat these students, even though no current research offers insight into how this should be carried out.

\section{ACKNOWLEDGMENTS}

This study was supported by the National Operating Committee on Standards for Athletic Equipment (NOCSAE).

\section{REFERENCES}

1. Guskiewicz KM, Weaver NL, Padua DA, Garrett WE Jr. Epidemiology of concussion in collegiate and high school football players. Am J Sports Med. 2000;28(5):643-650.

2. McCrea M, Hammeke T, Olsen G, Leo P, Guskiewicz K. Unreported concussion in high school football players: implications for prevention. Clin J Sport Med. 2004;14(1):13-17.

3. McCrory P, Meeuwisse WH, Aubry M, et al. Consensus statement on concussion in sport: the 4th International Conference on Concussion in Sport, Zurich, November 2012. J Athl Train. 2013;48(4):554-575.

4. Broglio SP, Cantu RC, Gioia GA, et al. National Athletic Trainers' Association position statement: management of sport concussion. $J$ Athl Train. 2014;49(2):245-265.

5. Notebaert AJ, Guskiewicz KM. Current trends in athletic training practice for concussion assessment and management. J Athl Train. 2005;40(4):320-325.

6. Covassin T, Elbin RJ III, Stiller-Ostrowski JL, Kontos AP. Immediate Post-Concussion Assessment and Cognitive Testing (ImPACT) practices of sports medicine professionals. J Athl Train. 2009;44(6):639-644.

7. Seidman LJ. Neuropsychological testing. Harvard Mental Health Letter. 1998;14(11):4-6.

8. Lynall RC, Laudner KG, Mihalik JP, Stanek JM. Concussionassessment and -management techniques used by athletic trainers. $J$ Athl Train. 2013;48(6):844-850.

9. Langlois JA, Rutland-Brown W, Wald MM. The epidemiology and impact of traumatic brain injury. J Head Trauma Rehabil. 2006; 21(5):375-378.

10. 2015-2016 high school athletics participation survey. The National Federation of State High School Associations Web site. http://www. 
nfhs.org/ParticipationStatistics/PDF/2015-16_Sports_Participation_ Survey.pdf. Updated 2016. Accessed January 10, 2017.

11. National Elementary and Secondary Enrollment Projection Model, 1972 through 2025. The National Center for Education Statistics Web site. https://nces.ed.gov/programs/digest/d15/tables/dt15_105. 20.asp?current=yes. Updated February 2016. Accessed January 10, 2017.

12. Benes F. The development of frontal cortex; the maturation of neurotransmitter systems and their interactions. In: Nelson C, Luciana M, eds. Handbook of Developmental Cognitive Neuroscience. Cambridge, MA: MIT Press; 2001:79-82.

13. Ursache A, Noble KG. Neurocognitive development in socioeconomic context: multiple mechanisms and implications for measuring socioeconomic status. Psychophysiology. 2016;53(1):71-82.

14. Schlaug G, Jancke L, Huang Y, Steinmetz H. In vivo evidence of structural brain asymmetry in musicians. Science. 1995;267(5198): 699-701.

15. Iverson GL, Lovell MR, Collins MW. Interpreting change on ImPACT following sport concussion. Clin Neuropsychol. 2003; 17(4):460-467.

16. Schatz P. Long-term test-retest reliability of baseline cognitive assessments using ImPACT. Am J Sports Med. 2010;38(1):47-53.

17. Grindel SH, Lovell MR, Collins MW. The assessment of sportrelated concussion: the evidence behind neuropsychological testing and management. Clin J Sport Med. 2001;11(3):134-143.

18. Schatz P, Sandel N. Sensitivity and specificity of the online version of ImPACT in high school and collegiate athletes. Am J Sports Med. 2013;41(2):321-326.

19. Vaughan CG, Gerst EH, Sady MD, Newman JB, Gioia GA. The relation between testing environment and baseline performance in child and adolescent concussion assessment. Am J Sports Med. 2014; 42(7):1716-1723.

20. Jensen AR. Clocking the Mind: Mental Chronometry and Individual Differences. Amsterdam, Netherlands: Elsevier; 2006.

21. Bruzi AT, Fialho JVAP, Fonseca FS, Ugrinowitsch H. Comparison of reaction time between basketball players, artistic gymnasts and non-athletes. Revista Brasileira de Ciencias do Esporte. 2013;35(2): 469-480.

22. Brisswalter J, Arcelin R, Audiffren M, Delignieres D. Influence of physical exercise on simple reaction time. Percept Mot Skills. 1997; 85(3 pt 1):1019-1027.

23. Tweit AH, Gollnick PD, Hearn GR. Effect of training program on total body reaction time of individuals of low fitness. Res $Q .1963$; 34(4):508-519.

24. Verma S, Mishra A, Singh A. Effect of long term exercise training on auditory and visual reaction time. Indian J Physiother Occup Ther. 2011;5(3):126-129.

25. Balko S, Borysiuk Z, Simonek J. The influence of different performance level of fencers on simple and choice reaction time.
Revista Brasileira de Cineantropometria \& Desempehno Humano. 2016;18(4):391-400.

26. Verbaugh L, Scherder EJA, van Lange PAM, Oosterlaan J. The key to success in elite athletes? Explicit and implicit motor learning in youth elite and non-elite soccer players. J Sport Sci. 2016;34(18): 1782-1790.

27. Williams LR, Walmsley A. Response amendment in fencing; differences between elite and novice subjects. Percept Mot Skills. 2000;91(1):131-142.

28. Kroshus E, Kubzansky LD, Goldman RE, Austin GS. Norms, athletic identity, and concussion symptom under-reporting among male collegiate ice hockey players: a prospective cohort study. Ann Behav Med. 2015;49(1):95-103.

29. Guest A, Schneider B. Adolescents' extracurricular participation in context: the mediating effects of schools, communities, and identity. Sociol Educ. 2003;79(2):89-109.

30. Brewer BW, Van Raalte JL, Linder DE. Athletic identity: Hercules' muscles or achilles heel? Int J Sport Psych. 1993;24(2):237-254.

31. Hughes R, Coakley J. Positive deviance among athletes: the implications of overconformity of the sport ethic. Sociol Sport J. 1991;8(4):307-325.

32. Nixon HL II. Accepting the risks of pain and injury in sport: mediated cultural influences of playing hurt. Sociol Sport J. 1993; 10(2):183-196.

33. Ryan ED, Kovacic CR. Pain tolerance and athletic participation. Percept Mot Skills. 1966;22(2):383-390.

34. Ho YC, Cheung MC, Chan AS. Music training improves verbal but not visual memory: cross-sectional and longitudinal explorations in children. Neuropsychology. 2003;17(3):439-450.

35. Kilgour A, Jakobson L, Cuddy L. Music training and rate of presentation as mediators of text and song recall. Mem Cognit. 2000; 28(5):700-710.

36. Dege F, Wehrum S, Stark R, Schwarzer G. The influence of two years of school music training in secondary school on visual and auditory memory. Eur J Dev Psychol. 2011;8(5):608-623.

37. Brandler S, Rammsayer T. Differences in mental abilities between musicians and nonmusicians. Psychol Music. 2003;31(2):123-138.

38. Chan AS, Ho YC, Cheung MC. Music training improves verbal memory. Nature. 1998;396(6707):128.

39. Hunt TN, Ferrara MS, Miller SL, Macciocchi S. The effect of effort on baseline neuropsychological test scores in high school football athletes. Arch Clin Neuropsychol. 2007;22(5):615-621.

40. Parker TM, Osternig LR, van Donkelaar P, Chou LS. Balance control during gait in athletes and non-athletes following concussion. Med Eng Phys. 2008;30(8):956-967.

41. Gur RC, Richard J, Calkins ME, et al. Age group sex differences in performance on a computerized neurocognitive battery in children age 8-21. Neuropsychology. 2012;26(2):251-265.

Address correspondence to Christopher P. Tomczyk, LAT, ATC, Department of Health Sciences and Kinesiology, Georgia Southern University, PO Box 8076, Statesboro, GA 30460. Address e-mail to cptom101@gmail.com. 